\title{
Patterns of hypnotic prescribing for residual insomnia and recurrence of major depressive disorder: a retrospective cohort study using a Japanese health insurance claims database
}

Kentaro Yamato ${ }^{1}$, Ken Inada ${ }^{2}$, Minori Enomoto ${ }^{3}$, Tatsuro Marumoto ${ }^{1}$, Masahiro Takeshima ${ }^{4}$ and Kazuo Mishima ${ }^{4,5,6^{*}}$

\begin{abstract}
Background: Major depressive disorder (MDD) is highly prevalent in Japan and frequently accompanied by insomnia that may persist even with MDD remission. Hypnotics are used for the pharmacological treatment of insomnia, but their influence on MDD recurrence or residual insomnia following MDD remission is unclear. This retrospective, longitudinal, cohort study utilized a large Japanese health insurance claims database to investigate patterns of hypnotic prescriptions among patients with MDD, and the influence of hypnotic prescription pattern on MDD recurrence.

Methods: Eligible patients (20-56 years) were those registered in the Japan Medical Data Center database between 1 January 2005 and 31 December 2018, and prescribed antidepressant and hypnotic therapy after being diagnosed with MDD. Patients who had ceased antidepressant therapy for $>180$ days were followed for 1 year to evaluate depression recurrence, as assessed using Kaplan-Meier estimates. Logistic regression modelling was used to analyze the effect of hypnotic prescription pattern on MDD recurrence.

(Continued on next page)
\end{abstract}

\footnotetext{
* Correspondence: mishima@med.akita-u.ac.jp

${ }^{4}$ Department of Neuropsychiatry, Akita University Graduate School of Medicine, Akita, Japan

${ }^{5}$ Department of Psychophysiology, National Institute of Mental Health, National Center of Neurology and Psychiatry, Tokyo, Japan

Full list of author information is available at the end of the article
}

C C The Author(s). 2021 Open Access This article is licensed under a Creative Commons Attribution 4.0 International License, which permits use, sharing, adaptation, distribution and reproduction in any medium or format, as long as you give appropriate credit to the original author(s) and the source, provide a link to the Creative Commons licence, and indicate if changes were made. The images or other third party material in this article are included in the article's Creative Commons licence, unless indicated otherwise in a credit line to the material. If material is not included in the article's Creative Commons licence and your intended use is not permitted by statutory regulation or exceeds the permitted use, you will need to obtain permission directly from the copyright holder. To view a copy of this licence, visit http://creativecommons.org/licenses/by/4.0/. The Creative Commons Public Domain Dedication waiver (http://creativecommons.org/publicdomain/zero/1.0/) applies to the data made available in this article, unless otherwise stated in a credit line to the data. 
(Continued from previous page)

Results: Of the 179,174 patients diagnosed with MDD who initiated antidepressant treatment between 1 January 2006 and 30 June 2017, complete prescription information was available for 2946 eligible patients who had been prescribed hypnotics. More patients were prescribed hypnotic monotherapy (70.8\%) than combination therapy (29.2\%). The most prescribed therapies were benzodiazepine monotherapy (26.2\%), non-benzodiazepine monotherapy (28.9\%), and combination therapy with two drugs (21.1\%). Among patients prescribed multiple hypnotics, concomitant prescriptions for anxiolytics, antipsychotics, mood stabilizers and sedative antidepressants were more common. The 1-year recurrence rate for MDD was approximately 20\%, irrespective of hypnotic monoversus combination therapy or class of hypnotic therapy. Being a spouse (odds ratio [OR], 1.44; 95\% confidence interval [Cl], 1.03-2.02) or other family member $(\mathrm{OR}, 1.46,95 \% \mathrm{Cl}, 0.99-2.16)$ of the insured individual, or being prescribed a sedative antidepressant $(\mathrm{OR}, 1.50,95 \% \mathrm{Cl}, 1.24-1.82)$ conferred higher odds of MDD recurrence within 1 year of completing antidepressant therapy.

Conclusions: Benzodiazepines are the most prescribed hypnotic among Japanese patients with MDD, though combination hypnotic therapy is routinely prescribed. Hypnotic prescription pattern does not appear to influence real-world MDD recurrence, though hypnotics should be appropriately prescribed given class differences in efficacy and safety.

Keywords: Major depressive disorder, Depression, Residual symptom, Insomnia, Recurrence, Japanese patients, Prescription pattern

\section{Background}

Major depressive disorder (MDD) is highly prevalent in Japan, with a 12 -month prevalence of $2.2-2.7 \%$ and lifetime prevalence of $6.1 \%[1,2]$. However, because only around $20 \%$ of Japanese patients with a mental disorder seek medical care [3], the prevalence of MDD may be higher than currently estimated. MDD is a leading cause of disability both globally and in Japan [4], and is associated with reduced quality of life, lower work productivity, and a substantial economic burden [5-7].

Treatment for MDD generally involves antidepressant therapy to achieve clinical remission and prevent relapse [8]. However, an estimated $20 \%$ of patients with MDD experience a recurrence within 6 months of recovery [8]. Relapse rates are particularly high among patients who discontinue treatment early [8], which may be particularly important among Japanese patients, in whom median treatment duration tends to be shorter than guideline recommendations [9].

Around one-quarter of patients with MDD have residual symptoms following remission [8], such as depressed mood, anxiety, and insomnia [10]. Importantly, residual symptoms are associated with MDD relapse and recurrence $[10,11]$. Insomnia, in particular, is a common residual symptom experienced by more than $50 \%$ of patients, which, in addition to increasing the risk of MDD relapse, is associated with reduced quality of life [10, 12].

Hypnotic therapies, including benzodiazepine and non-benzodiazepine drugs, sedative-hypnotic drugs (such as sedating antidepressants), melatonin, and orexin-receptor antagonists, are used in the pharmacological treatment of insomnia $[13,14]$. Hypnotic therapies are commonly co-prescribed with antidepressants to manage depression-associated insomnia [13, 15]. While hypnotic therapies can improve sleep for patients with depression-associated residual insomnia [16, 17], they have also been associated with an increased risk of developing a psychiatric disorder [18]. High doses of hypnotics have also been linked to antidepressant refractoriness and worse depression outcomes [19].

Therefore, this retrospective cohort study aimed to investigate real-world patterns of hypnotic prescribing among Japanese patients with MDD, and the influence of hypnotic prescription pattern on MDD recurrence, by interrogating the Japan Medical Data Center (JMDC) Inc. insurance claims database, which comprises accumulated health insurance receipt data (inpatient, outpatient, dispensing) from multiple health insurance associations in Japan for $>7$ million individuals. The database also contains patients' medical examination data including diagnosis and treatment information, enabling longitudinal tracking of patients across different healthcare providers.

\section{Methods}

\section{Data sources, study population and cohort selection}

We retrospectively analyzed anonymized health insurance claims data collated in the JMDC Inc. database (system version: Netezza N2002 010 7.1.0.4.P2; Tokyo, Japan), which comprises accumulated receipts (inpatient, outpatient and dispensing) and medical examination data received from multiple health insurance associations relating to $7,175,048$ people in Japan, between 1 January 2005 and 31 December 2018. Eligible patients received a diagnosis of primary or recurrent MDD (World Health Organization's International Statistical 
Classification of Diseases and Related Health Problems [10th ed.; ICD-10] codes F32 or F33, respectively) and were prescribed antidepressant therapy, defined as a selective serotonin reuptake inhibitor (SSRI), serotoninnoradrenaline reuptake inhibitor (SNRI), noradrenergic or specific serotonergic antidepressant, or tricyclic antidepressant (World Health Organization's Anatomical Therapeutic Chemical [ATC] codes N06A4, N06A5, N06A9 and N06A9, respectively) and a hypnotic (nonbarbiturate, monotherapy; ATC code N05B1) between 1 January 2006 and 30 June 2017 (the study enrollment period; Fig. 1).

The index date was the date on which all antidepressant treatment was expected to be completed following dispensing of the final antidepressant prescription. Eligible patients must have had $\geq 1$ period of $\geq 180$ days without antidepressant treatment after the index date (the treatment completion period). Patients were also required to be aged $\geq 20$ years at the time of initiating antidepressant therapy and $<65$ years within the entire observable period from starting antidepressant therapy of up to 540 days from the index date. Patients must have had continuous health insurance enrollment for $\geq 12$ months prior to the earliest diagnosis of depression during the study enrollment period.

Patients were excluded from the study if they had been diagnosed with MDD and not prescribed an antidepressant, or prescribed antidepressants or hypnotics without a diagnosis of MDD during the baseline period (365 days prior to first being diagnosed with MDD and prescribed an antidepressant).

\section{Variables}

Outcomes assessed in this exploratory study were the pattern of hypnotic prescription for depressionassociated insomnia, and the impact of hypnotic prescription patterns on time to MDD relapse, defined as the reissue of a prescription for antidepressant therapy after a >180-day period without antidepressant therapy, in combination with a hypnotic for residual insomnia.

Predictor variables included patients' demographic characteristics, insurance enrolment category (insured, spouse, or other dependent family member), prior or concomitant diagnosis of other psychiatric disorders (anxiety disorder, autism, schizophrenia, attention deficit hyperactivity disorder, bipolar disorder [ICD-10 codes: F20-F31, F40-F45, F48, F84.0, F90.0]), prior or concomitant use of other medications, (ATC codes: A01A-, A02B1, A02B2, A07E2, C01A1, C01B-, C02A1, C02A2, C02B2, C02C-, C02D-, C03A2, C03A3, C05A1, C06X-, C07A-, D05X-, D06A-, D07A-, D07B1, D07B4, D11A-, G01A1, G01A2, G03B-, G03C-, G03D-, G03E-, G03F-, G03G-, G03J-, G03X-, G04A2, H01A-, H02A1, H02A2, H02B-, H03A-, J01A-, J01C1, J01C2, J01E-, J01X9, J03A-, J04A1, J05B3, J05D1, J08B-, L01C1, L01D-, L01X9, L03B1, L03B2, L03B3, L04X-, M01A1, M02A-, M03B-, N01A2, N02B-, N03A-, N04A-, N05A9, N05BA, N05BA01-N05BA03, N05BA05, N05BA06, N05BA08, N05BA09, N05BA11, N05BA12, N05BA17-N05BA19, N05BA21-N05BA23, N05BB01, N05BE, N05B3, N07A-, N07E-, N07X-, R01A1, R02A-, R03D1, R03F1, R03L3, R05A-, R06A-, S01A-, S01B-, S01E1, S01E2, S01R-, S02C-, S03B-, S03C-), prescription for sedative

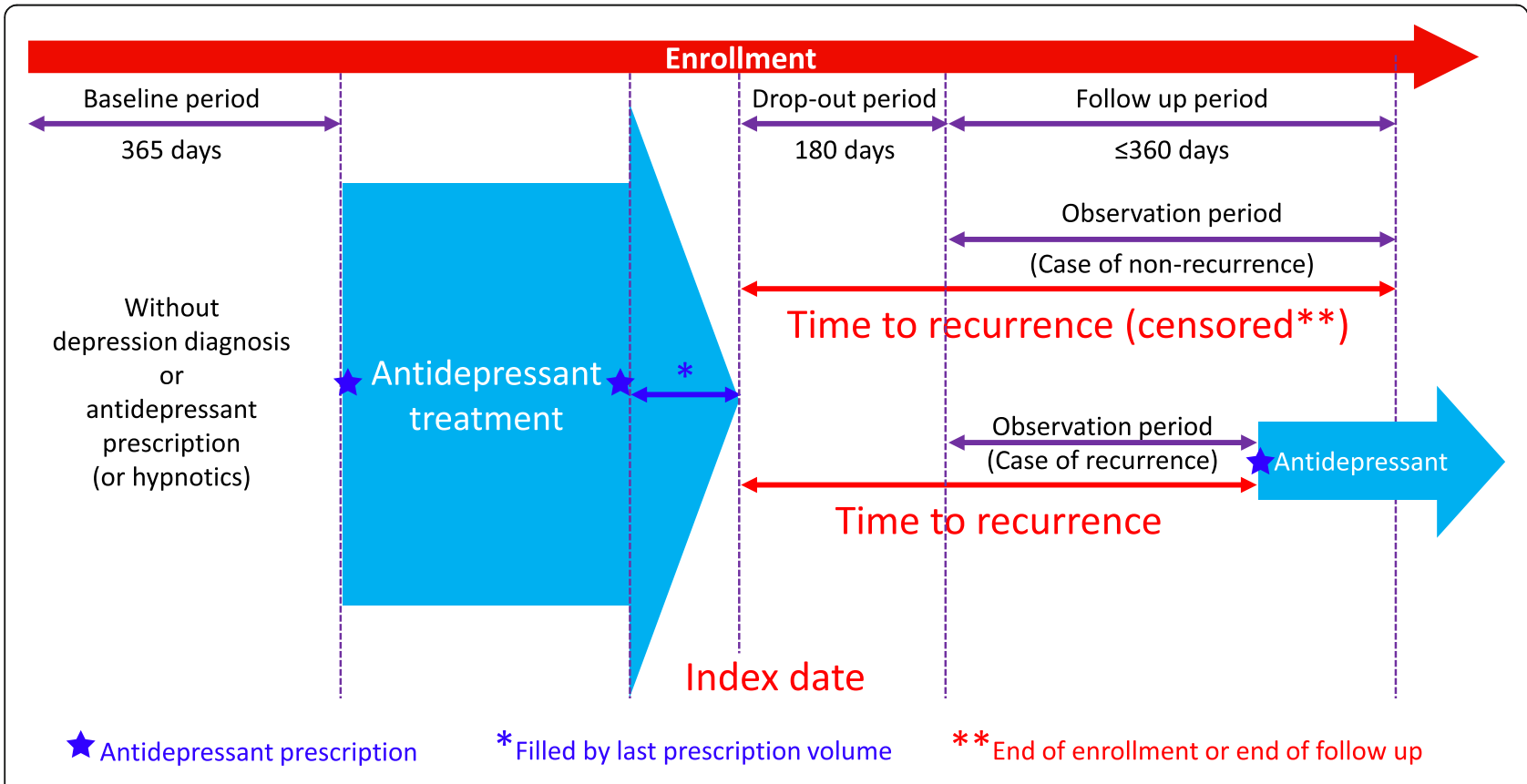

Fig. 1 Study design 
antidepressant medications (mirtazapine, trazodone hydrochloride, mianserin hydrochloride), antidepressant dosage at the end of treatment (less than or greater than the recommended maximum dose), the daily imipramine-equivalent dose of antidepressant $(<75 \mathrm{mg} /$ day, $\geq 75$ and $<150 \mathrm{mg} /$ day, or $\geq 150 \mathrm{mg} /$ day) [20]. Prior disorders were those diagnosed $<12$ months before the diagnosis and treatment of depression. Concomitant medications were those prescribed $\leq 6$ months before the follow-up period.

Patients' hypnotic prescription pattern was categorized as benzodiazepine monotherapy, non-benzodiazepine monotherapy, melatonin receptor agonists, orexin receptor antagonists, two agents combined, three agents combined, or $\geq 4$ drugs.

\section{Statistical analysis}

Patient demographics, clinical characteristics and study variables were summarized using descriptive statistics (mean, standard deviation [SD], median, interquartile range $[I Q R])$. If the date of prescription was missing from the receipt, the last day of the month in which the doctor's office visit occurred was used. If the dose of medication was missing, the data point was removed from the analysis.

The impact of hypnotic prescribing patterns on MDD recurrence during the follow-up period was assessed using Kaplan-Meier estimates.

Logistic regression modelling was used to analyze the effect of hypnotic prescription pattern on MDD relapse, with time to MDD relapse as the dependent variable, hypnotic prescription pattern as an explanatory variable, and demographic/clinical characteristics as independent variables. Adjusted odds ratios (OR) and 95\% confidence intervals $(\mathrm{CI})$ were calculated using a Cox proportional hazards model. A stepwise method was used to select variables to be included in the final model, using an inclusion significance level of $<0.1$ and exclusion significance level of $\geq 0.05$. A final logistic regression model was developed using age and gender (mandatory variables) and all significant predictors of MDD relapse, with the 1-year recurrence rate as the dependent variable and residual insomnia as an explanatory variable.

All statistical analyses were conducted using SAS version 9.4 (TS1M4; SAS Institute Japan Ltd., Tokyo, Japan).

\section{Results}

Of the 179,174 patients diagnosed with MDD who initiated antidepressant treatment between 1 January 2006 and 30 June 2017, 36,192 patients fulfilled the eligibility criteria for this study (Fig. 2). Of those, $\geq 1$-year followup data was available for 30,381 patients, of whom 4166 had been prescribed hypnotics for residual insomnia during the follow-up period. Among those prescribed hypnotics for residual insomnia, complete prescription information was available for 2946 patients (the total analyzed population).

\section{Patient demographics and treatment pattern}

Patients in the total analyzed population $(n=2946)$ were mostly male $(62.8 \%)$ and aged $\geq 40$ years $(58.8 \%)$, with a mean (SD) age of 41.6 (10.2) years (Table 1). Approximately three-quarters of patients were insured employees (74.2\%). All other patients were the spouse $(19.2 \%)$ or another family member $(6.6 \%)$ of the insured individual. While anxiety disorder (16.6\%) was the most prevalent psychiatric condition prior to MDD diagnosis, bipolar disorder (85.4\%), anxiety disorder (40.7\%) and schizophrenia $(15.8 \%)$ were the most commonly comorbid psychiatric disorders at the time of diagnosis with MDD. Steroidal and hormonal drugs (48.8\%) and anxiolytics $(18.8 \%)$ were the most commonly prescribed medications prior to MDD diagnosis. At the time of diagnosis with MDD, $37.9 \%$ of patients were prescribed steroidal and hormonal drugs and concomitant anxiolytics, antipsychotics, and mood stabilizers were prescribed to 48.4, 20.6 and $7.3 \%$ of patients, respectively. Mirtazapine (20.4\%) was the most frequently prescribed sedative antidepressant, followed by trazodone hydrochloride (12.2\%), and mianserin hydrochloride $(3.2 \%)$. At the end of treatment, almost all patients (93\%) were receiving less than the maximum recommended dose of antidepressant.

The vast majority of patients (70.8\%) were prescribed hypnotic monotherapy rather than combination therapy comprising $\geq 2$ drugs (29.2\%). Most prescriptions were for benzodiazepine monotherapy (36.2\%) and nonbenzodiazepine monotherapy (28.9\%), followed by combination therapy with two drugs (21.1\%). Patient demographics and clinical characteristics among hypnotic prescription subgroups were generally comparable to the total analyzed population regarding age, male sex, insurance type, prior and concomitant psychiatric disorders and medications, sedative antidepressant prescriptions, and antidepressant dosage at the end of treatment. Similarly, patient demographics and clinical characteristics were generally comparable between patients prescribed monotherapy versus combination therapy, except that a greater proportion of patients prescribed hypnotic monotherapy were aged $\geq 40$ years $(61.2 \%$ vs $52.9 \%)$, and more patients prescribed combination hypnotic therapy had comorbid schizophrenia (24.1\% vs $12.4 \%)$. Furthermore, concomitant prescriptions for antipsychotics and mood stabilizers were more common among patients prescribed combination hypnotic therapy (31.4 and $13.5 \%$ ) versus monotherapy (16.1 and $4.7 \%)$. Specifically, the rate of concomitant antipsychotic prescriptions was 


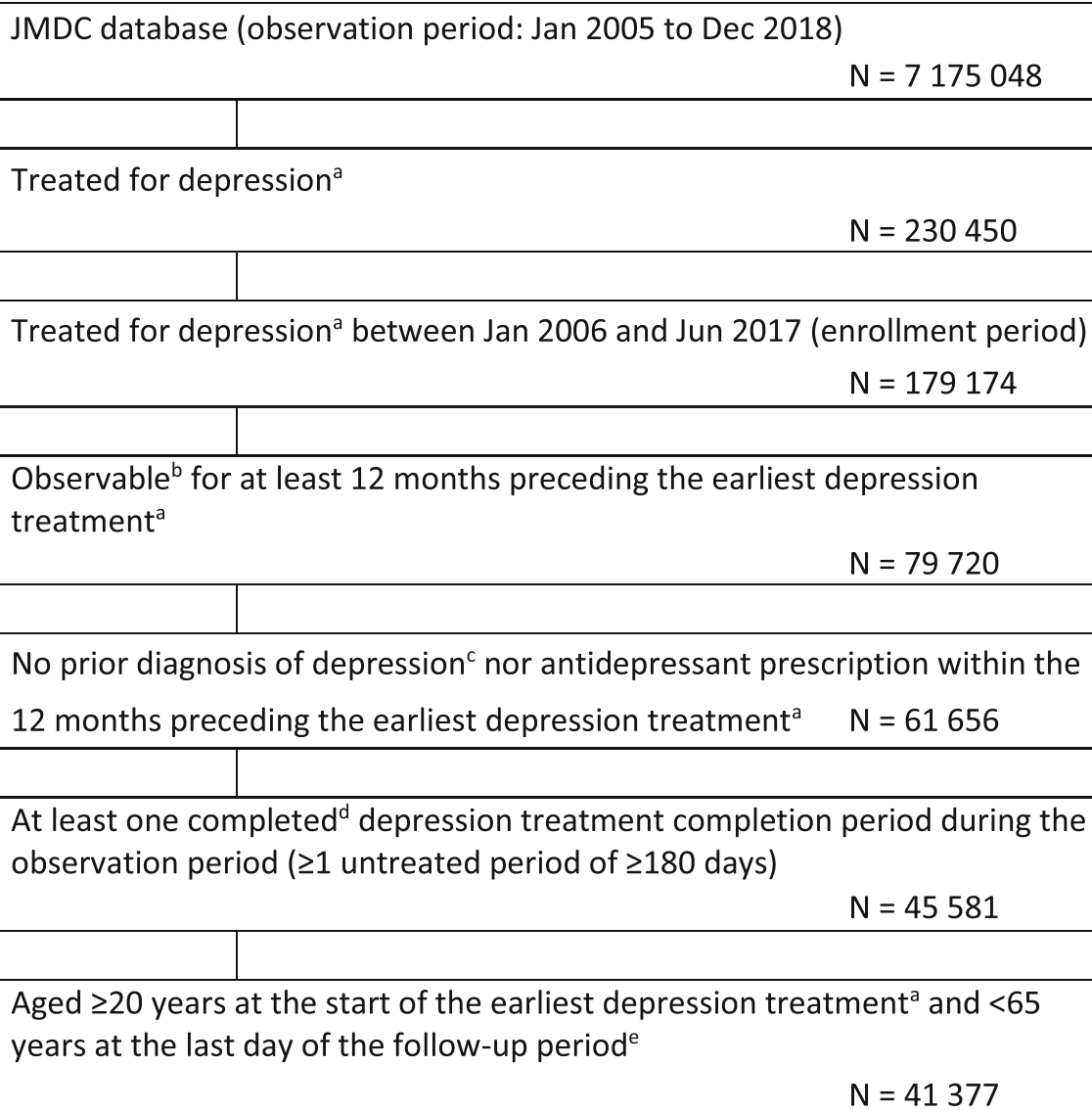

\begin{tabular}{|l|ll|}
\hline $\begin{array}{l}\text { Exclusion } \\
\text { Prescription of hypnotics within the } 12 \text { months preceding the earliest } \\
\text { treatment }^{\mathrm{a}}\end{array}$ & $\mathrm{N}=5168$ \\
Not observable $^{\mathrm{b}}$ at the start of the follow-up period & $\mathrm{N}=17$ \\
\hline
\end{tabular}

Full analysis set

$N=36192$

Observable $^{b}$ for at least 360 days following the start of the follow-up period ${ }^{\mathrm{e}}$

(analysis set for 1-year recurrence rate)

$\mathrm{N}=30381$

Patients with residual insomnia

$\mathrm{N}=4166$

Patients without residual insomnia

Total analyzed population

$\mathrm{N}=2946$

Fig. 2 (See legend on next page.) 
(See figure on previous page.)

Fig. 2 Flow diagram of study participants. ${ }^{a}$ A diagnosis of depression accompanied by prescription of antidepressants; ${ }^{b}$ Has continuous health insurance enrollment; ${ }^{c}$ Excluding the month immediately preceding the earliest treatment for diagnosis only; ${ }^{d} H a d$ no antidepressant treatment for $\geq 180$ continuous days; ${ }^{\text {Up }}$ to 360 days following the last day of the first untreated period of 180 continuous days. JMDC; Japan Medical Data Center

28.6, 35.5 and $46.5 \%$ among patients prescribed two-, three- or $\geq 4$-drug combination hypnotic therapy, respectively, versus $20.6 \%$ overall. Similarly, while $7.3 \%$ of patients were prescribed mood stabilizers overall, combination hypnotic therapies were associated with mood stabilizer prescription rates of $11.7,15.7$ and $23.9 \%$, respectively. Concomitant prescriptions for anxiolytics also tended to be higher among patients prescribed threedrug (57.8\%) or $\geq 4$-drug (62.0\%) combination hypnotic therapy. Prescriptions for sedative antidepressants also tended to be higher among patients prescribed two(24.0\%), three- $(25.9 \%)$ or $\leq 4$-drug (28.2\%) combination hypnotic therapies; a similar pattern was observed with trazodone hydrochloride and mianserin hydrochloride prescriptions.

Overall, mean antidepressant dosage was notably higher among patients prescribed combination therapy than monotherapy (83.1 vs $70.7 \mathrm{mg} /$ day). Specifically, mean antidepressant dosage was higher among patients prescribed two- (82.5 imipramine-equivalent $\mathrm{mg} /$ day), three- (87.7 imipramine-equivalent $\mathrm{mg} /$ day), or $\geq 4$-drug (77.4 imipramine-equivalent $\mathrm{mg} /$ day) combination hypnotic therapy compared with the total analyzed population (74.3 imipramine-equivalent $\mathrm{mg} /$ day).

\section{Effect of hypnotic prescription pattern on MDD recurrence}

There were no differences in MDD recurrence rates between patients prescribed monotherapy versus combination therapy for residual insomnia, or according to class of hypnotic prescribed (Fig. 3). Specifically, 1-year recurrence rates were $20 \%(n=213)$ with benzodiazepine, $17.2 \%(n=146)$ with non-benzodiazepine, $18.4 \%(\mathrm{n}=14)$ with melatonin receptor agonist and $18.1 \%(n=17)$ with orexin receptor antagonist therapy. Recurrence rates were $18.7 \%(n=390)$ with hypnotic monotherapy versus $23.3 \%(n=200)$ with combination treatment. Given the intersecting natures of the Kaplan-Meier curves (Fig. 3), we did not conduct log-rank and Wilcoxon tests to compare recurrence rates between groups.

\section{Factors associated with MDD recurrence}

Among patients prescribed hypnotic therapy for depression-associated residual insomnia, the odds of MDD recurrence within 1 year of completing antidepressant therapy were higher among those who were a spouse (OR, 1.44; 95\% CI, 1.03-2.02) or other family member (OR, 1.46; 95\% CI, 0.99-2.16) of the insured individual, and those who were prescribed a sedative antidepressant (OR, 1.50, 95\% CI, 1.24-1.82; Table 2), but lower among females (OR, 0.67; 95\% CI, 0.50-0.88). In contrast, prescription pattern did not influence the odds of MDD recurrence.

\section{Discussion}

This retrospective study utilized a large health insurance claims database to explore hypnotic prescription patterns and depression recurrence among Japanese patients with MDD. The findings illustrate that among patients with MDD prescribed hypnotic therapy, most were prescribed monotherapy after ceasing antidepressant therapy, predominantly benzodiazepines, while about one-third of patients were prescribed combination hypnotic therapy comprising $\geq 2$ drugs. Importantly, concomitant prescriptions for anxiolytics, antipsychotics, mood stabilizers and sedative antidepressants were more common among patients prescribed multiple hypnotic therapies.

In patients prescribed combination hypnotic therapy, antidepressant dosages were substantially higher compared with patients prescribed monotherapy, which may reflect greater severity of depression or antidepressantresistant depression in patients prescribed combination therapy. While severe depression is considered a risk factor for MDD recurrence [8, 21], there was no difference in recurrence rates according to hypnotic prescription in this study. Accordingly, hypnotic combination therapy may have prevented MDD recurrence in patients with severe depression.

While a previous study reported a significantly higher risk of depressive disorders over 6 years with long-term sedative-hypnotic prescriptions, including benzodiazepines in patients with insomnia [18], the current findings suggest depression recurrence among Japanese patients is unaffected by hypnotic prescription over a shorter, 1year period. Likewise, no differences in MDD recurrence rates was observed between patients prescribed hypnotic monotherapy versus combination therapy, or between classes of hypnotic therapy. However, it must be noted that sedatives and hypnotics are considered to be a short-term solution for managing insomnia, and this study has investigated their use in this context.

These findings confirm the relatively high rates of psychotropic polypharmacy in Japan [9, 22, 23]. Although Japanese treatment guidelines for MDD recommend antidepressant monotherapy, in reality around three- 
Table 1 Patient demographics and clinical characteristics

\begin{tabular}{|c|c|c|c|c|c|c|c|c|c|c|}
\hline \multirow[t]{2}{*}{ Variable } & \multirow{2}{*}{$\begin{array}{l}\text { All } \\
(N= \\
2946)\end{array}$} & \multicolumn{5}{|l|}{ Monotherapy } & \multicolumn{4}{|c|}{ Combination therapy } \\
\hline & & $\begin{array}{l}\text { Benzodiazepine } \\
(n=1067)\end{array}$ & $\begin{array}{l}\text { Non- } \\
\text { benzodiazepine } \\
(n=850)\end{array}$ & $\begin{array}{l}\text { Melatonin } \\
\text { receptor } \\
\text { agonist }(n= \\
76)\end{array}$ & $\begin{array}{l}\text { Orexin } \\
\text { receptor } \\
\text { antagonist } \\
(n=94)\end{array}$ & $\begin{array}{l}\text { Total } \\
(n= \\
2087)\end{array}$ & $\begin{array}{l}2 \\
\text { drugs } \\
(n= \\
622)\end{array}$ & $\begin{array}{l}3 \\
\text { drugs } \\
(n= \\
166)\end{array}$ & $\begin{array}{l}\geq 4 \\
\text { drugs } \\
(n= \\
71)\end{array}$ & $\begin{array}{l}\text { Total } \\
(n= \\
859)\end{array}$ \\
\hline $\begin{array}{l}\text { Age, years, } \\
\text { mean } \pm \text { SD }\end{array}$ & $\begin{array}{l}41.6 \pm \\
10.2\end{array}$ & $42.4 \pm 9.9$ & $42.2 \pm 10.0$ & $38.3 \pm 11.2$ & $42.5 \pm 11.4$ & $\begin{array}{l}42.2 \pm \\
10.1\end{array}$ & $\begin{array}{l}40.5 \pm \\
10.4\end{array}$ & $\begin{array}{l}38.9 \pm \\
10.1\end{array}$ & $\begin{array}{l}38.9 \pm \\
9.1\end{array}$ & $\begin{array}{l}40.1 \pm \\
10.2\end{array}$ \\
\hline$<40$ years, $\mathrm{n}(\%)$ & $\begin{array}{l}1214 \\
(41.2)\end{array}$ & $404(37.9)$ & $328(38.6)$ & $37(48.7)$ & $40(42.6)$ & $\begin{array}{l}809 \\
(38.8)\end{array}$ & $\begin{array}{l}284 \\
(45.7)\end{array}$ & $\begin{array}{l}84 \\
(50.6)\end{array}$ & $\begin{array}{l}37 \\
(52.1)\end{array}$ & $\begin{array}{l}405 \\
(47.1)\end{array}$ \\
\hline$\geq 40$ years, $n(\%)$ & $\begin{array}{l}1732 \\
(58.8)\end{array}$ & $663(62.1)$ & $522(61.4)$ & $39(51.3)$ & $54(57.4)$ & $\begin{array}{l}1278 \\
(61.2)\end{array}$ & $\begin{array}{l}338 \\
(54.3)\end{array}$ & $\begin{array}{l}82 \\
(49.4)\end{array}$ & $\begin{array}{l}34 \\
(47.9)\end{array}$ & $\begin{array}{l}454 \\
(52.9)\end{array}$ \\
\hline \multicolumn{11}{|l|}{ Sex, n (\%) } \\
\hline Male & $\begin{array}{l}1850 \\
(62.8)\end{array}$ & $693(64.9)$ & $507(59.6)$ & $44(57.9)$ & $51(54.3)$ & $\begin{array}{l}1295 \\
(62.1)\end{array}$ & $\begin{array}{l}403 \\
(64.8)\end{array}$ & $\begin{array}{l}105 \\
(63.3)\end{array}$ & $\begin{array}{l}47 \\
(66.2)\end{array}$ & $\begin{array}{l}555 \\
(64.6)\end{array}$ \\
\hline \multicolumn{11}{|c|}{ Insured or dependent, $\mathrm{n}(\%)$} \\
\hline Insured & $\begin{array}{l}2186 \\
(74.2)\end{array}$ & $805(75.4)$ & $619(72.8)$ & $49(64.5)$ & $68(72.3)$ & $\begin{array}{l}1541 \\
(73.8)\end{array}$ & $\begin{array}{l}468 \\
(75.2)\end{array}$ & $\begin{array}{l}124 \\
(74.7)\end{array}$ & $\begin{array}{l}53 \\
(74.6)\end{array}$ & $\begin{array}{l}645 \\
(75.1)\end{array}$ \\
\hline Spouse & $\begin{array}{l}567 \\
(19.2)\end{array}$ & $201(18.8)$ & $187(22.0)$ & $14(18.4)$ & $25(26.6)$ & $\begin{array}{l}427 \\
(20.5)\end{array}$ & $\begin{array}{l}103 \\
(16.6)\end{array}$ & $\begin{array}{l}24 \\
(14.5)\end{array}$ & $\begin{array}{l}13 \\
(18.3)\end{array}$ & $\begin{array}{l}140 \\
(16.3)\end{array}$ \\
\hline $\begin{array}{l}\text { Other family } \\
\text { member }\end{array}$ & $\begin{array}{l}193 \\
(6.6)\end{array}$ & $61(5.7)$ & $44(5.2)$ & $13(17.1)$ & $1(1.1)$ & $\begin{array}{l}119 \\
(5.7)\end{array}$ & $51(8.2)$ & $\begin{array}{l}18 \\
(10.8)\end{array}$ & $5(7.0)$ & $\begin{array}{l}74 \\
(8.6)\end{array}$ \\
\hline \multicolumn{11}{|c|}{ History of psychiatric disorders ${ }^{b}, n(\%)$} \\
\hline Anxiety disorder & $\begin{array}{l}489 \\
(16.6)\end{array}$ & $179(16.8)$ & $155(18.2)$ & $14(18.4)$ & $16(17.0)$ & $\begin{array}{l}364 \\
(17.4)\end{array}$ & $\begin{array}{l}89 \\
(14.3)\end{array}$ & $\begin{array}{l}23 \\
(13.9)\end{array}$ & $\begin{array}{l}13 \\
(18.3)\end{array}$ & $\begin{array}{l}125 \\
(14.6)\end{array}$ \\
\hline Bipolar disorder & $\begin{array}{l}14 \\
(0.5)\end{array}$ & $3(0.3)$ & $8(0.9)$ & $1(1.3)$ & 0 & $\begin{array}{l}12 \\
(0.6)\end{array}$ & $2(0.3)$ & $0(0)$ & 0 & $2(0.2)$ \\
\hline Schizophrenia & $\begin{array}{l}40 \\
(1.4)\end{array}$ & $18(1.7)$ & $5(0.6)$ & $1(1.3)$ & 0 & $\begin{array}{l}24 \\
(1.1)\end{array}$ & $10(1.6)$ & $6(3.6)$ & 0 & $\begin{array}{l}16 \\
(1.9)\end{array}$ \\
\hline ADHD & $2(0.1)$ & 0 & 0 & 0 & 0 & 0 & $2(0.3)$ & 0 & 0 & $2(0.2)$ \\
\hline Autism & $1(0.0)$ & 0 & 0 & 0 & 0 & 0 & $1(0.2)$ & 0 & 0 & $1(0.1)$ \\
\hline \multicolumn{11}{|c|}{ Comorbid psychiatric disorders ${ }^{c}, \mathrm{n}(\%)$} \\
\hline Anxiety disorder & $\begin{array}{l}1200 \\
(40.7)\end{array}$ & $416(39.0)$ & $343(40.4)$ & $36(47.7)$ & $45(47.9)$ & $\begin{array}{l}840 \\
(40.2)\end{array}$ & $\begin{array}{l}263 \\
(42.3)\end{array}$ & $\begin{array}{l}62 \\
(37.3)\end{array}$ & $\begin{array}{l}35 \\
(49.3)\end{array}$ & $\begin{array}{l}360 \\
(41.9)\end{array}$ \\
\hline Bipolar disorder & $\begin{array}{l}509 \\
(17.3)\end{array}$ & $167(15.7)$ & $78(9.2)$ & $10(13.2)$ & $13(13.8)$ & $\begin{array}{l}268 \\
(12.8)\end{array}$ & $\begin{array}{l}166 \\
(26.7)\end{array}$ & $\begin{array}{l}47 \\
(28.3)\end{array}$ & $\begin{array}{l}28 \\
(39.4)\end{array}$ & $\begin{array}{l}241 \\
(28.1)\end{array}$ \\
\hline Schizophrenia & $\begin{array}{l}466 \\
(15.8)\end{array}$ & $179(16.8)$ & $61(7.2)$ & $6(7.9)$ & $13(13.8)$ & $\begin{array}{l}259 \\
(12.4)\end{array}$ & $\begin{array}{l}138 \\
(22.2)\end{array}$ & $\begin{array}{l}47 \\
(28.3)\end{array}$ & $\begin{array}{l}22 \\
(31.0)\end{array}$ & $\begin{array}{l}207 \\
(24.1)\end{array}$ \\
\hline ADHD & $\begin{array}{l}48 \\
(1.6)\end{array}$ & $16(1.5)$ & $4(0.5)$ & $2(2.6)$ & $3(3.2)$ & $\begin{array}{l}25 \\
(1.2)\end{array}$ & $13(2.1)$ & $9(5.4)$ & $1(1.4)$ & $\begin{array}{l}23 \\
(2.7)\end{array}$ \\
\hline Autism & $2(0.1)$ & 0 & 0 & 0 & 0 & 0 & $2(0.3)$ & 0 & 0 & $2(0.2)$ \\
\hline \multicolumn{11}{|c|}{ History of medication at baseline ${ }^{b}, \mathrm{n}(\%)$} \\
\hline $\begin{array}{l}\text { Steroid, hormonal } \\
\text { drug }\end{array}$ & $\begin{array}{l}1437 \\
(48.8)\end{array}$ & $501(47.0)$ & $452(53.2)$ & $36(47.4)$ & $53(56.4)$ & $\begin{array}{l}1042 \\
(49.9)\end{array}$ & $\begin{array}{l}293 \\
(47.1)\end{array}$ & $\begin{array}{l}73 \\
(44.0)\end{array}$ & $\begin{array}{l}29 \\
(40.8)\end{array}$ & $\begin{array}{l}395 \\
(46.0)\end{array}$ \\
\hline Anxiolytics & $\begin{array}{l}555 \\
(18.8)\end{array}$ & $201(18.8)$ & $176(20.7)$ & 15 (19.7) & $18(19.1)$ & $\begin{array}{l}410 \\
(19.6)\end{array}$ & $\begin{array}{l}101 \\
(16.2)\end{array}$ & $\begin{array}{l}31 \\
(18.7)\end{array}$ & $\begin{array}{l}13 \\
(18.3)\end{array}$ & $\begin{array}{l}145 \\
(16.9)\end{array}$ \\
\hline $\begin{array}{l}\text { Leukotriene } \\
\text { receptor } \\
\text { antagonists }\end{array}$ & $\begin{array}{l}276 \\
(9.4)\end{array}$ & $96(9.0)$ & $82(9.6)$ & $6(7.9)$ & $9(9.6)$ & $\begin{array}{l}193 \\
(9.2)\end{array}$ & $54(8.7)$ & $\begin{array}{l}21 \\
(12.7)\end{array}$ & $8(11.3)$ & $\begin{array}{l}83 \\
(9.7)\end{array}$ \\
\hline $\begin{array}{l}\text { Neurological } \\
\text { medication, } \\
\text { psychotropic drug }\end{array}$ & $\begin{array}{l}198 \\
(6.7)\end{array}$ & $66(6.2)$ & $60(7.1)$ & $2(2.6)$ & $5(5.3)$ & $\begin{array}{l}133 \\
(6.4)\end{array}$ & $43(6.9)$ & $15(9.0)$ & $7(9.9)$ & $\begin{array}{l}65 \\
(7.6)\end{array}$ \\
\hline $\begin{array}{l}\text { Analgesic, anti- } \\
\text { inflammatory }\end{array}$ & $\begin{array}{l}187 \\
(6.3)\end{array}$ & $67(6.3)$ & $55(6.5)$ & $1(1.3)$ & $8(8.5)$ & $\begin{array}{l}131 \\
(6.3)\end{array}$ & $44(7.1)$ & $11(6.6)$ & $1(1.4)$ & $\begin{array}{l}56 \\
(6.5)\end{array}$ \\
\hline Antibacterial and/ & 179 & $56(5.2)$ & $53(6.2)$ & $5(6.6)$ & $4(4.3)$ & 118 & $41(6.6)$ & $15(9.0)$ & $5(7.0)$ & 61 \\
\hline
\end{tabular}


Table 1 Patient demographics and clinical characteristics (Continued)

\begin{tabular}{|c|c|c|c|c|c|c|c|c|c|c|}
\hline \multirow[t]{2}{*}{ Variable } & \multirow{2}{*}{$\begin{array}{l}\text { All } \\
(N= \\
2946)\end{array}$} & \multicolumn{5}{|l|}{ Monotherapy } & \multicolumn{4}{|c|}{ Combination therapy } \\
\hline & & $\begin{array}{l}\text { Benzodiazepine } \\
(n=1067)\end{array}$ & $\begin{array}{l}\text { Non- } \\
\text { benzodiazepine } \\
(n=850)\end{array}$ & $\begin{array}{l}\text { Melatonin } \\
\text { receptor } \\
\text { agonist }(n= \\
76)\end{array}$ & $\begin{array}{l}\text { Orexin } \\
\text { receptor } \\
\text { antagonist } \\
(n=94)\end{array}$ & $\begin{array}{l}\text { Total } \\
(n= \\
2087)\end{array}$ & $\begin{array}{l}2 \\
\text { drugs } \\
(n= \\
622)\end{array}$ & $\begin{array}{l}\begin{array}{l}3 \\
\text { drugs } \\
(n= \\
166)\end{array} \\
\end{array}$ & $\begin{array}{l}\geq 4 \\
\text { drugs } \\
(n= \\
71)\end{array}$ & $\begin{array}{l}\text { Total } \\
(n= \\
859)\end{array}$ \\
\hline or antifungal & $(6.1)$ & & & & & $(5.7)$ & & & & $(7.1)$ \\
\hline $\begin{array}{l}\text { Antihypertensive, } \\
\text { cardiotonic }\end{array}$ & $\begin{array}{l}52 \\
(1.8)\end{array}$ & $14(1.3)$ & $22(2.6)$ & $3(3.9)$ & $1(1.1)$ & $\begin{array}{l}40 \\
(1.9)\end{array}$ & $8(1.3)$ & $4(2.4)$ & 0 & $\begin{array}{l}12 \\
(1.4)\end{array}$ \\
\hline Antipsychotic & $\begin{array}{l}49 \\
(1.7)\end{array}$ & $18(1.7)$ & $13(1.5)$ & $1(1.3)$ & 0 & $\begin{array}{l}32 \\
(1.5)\end{array}$ & $10(1.6)$ & $7(4.2)$ & 0 & $\begin{array}{l}17 \\
(2.0)\end{array}$ \\
\hline $\begin{array}{l}\text { Antismoking } \\
\text { product }\end{array}$ & $\begin{array}{l}21 \\
(0.7)\end{array}$ & $9(0.8)$ & $5(0.6)$ & 0 & 0 & $\begin{array}{l}14 \\
(0.7)\end{array}$ & $7(1.1)$ & 0 & 0 & $7(0.8)$ \\
\hline Antineoplastic & $7(0.2)$ & $2(0.2)$ & $3(0.4)$ & $1(1.3)$ & $1(1.1)$ & $7(0.3)$ & 0 & 0 & 0 & 0 \\
\hline Mood stabilizer & $2(0.1)$ & $1(0.1)$ & $1(0.1)$ & 0 & 0 & $2(0.1)$ & 0 & 0 & 0 & 0 \\
\hline Immunostimulant & $2(0.1)$ & $2(0.2)$ & 0 & 0 & 0 & $2(0.1)$ & 0 & 0 & 0 & 0 \\
\hline $\begin{array}{l}\text { Centrally-acting } \\
\text { anti-obesity } \\
\text { product }\end{array}$ & $1(0)$ & 0 & 0 & 0 & 0 & 0 & $1(0.2)$ & 0 & 0 & $1(0.1)$ \\
\hline Other & $\begin{array}{l}324 \\
(11.0)\end{array}$ & $124(11.6)$ & $92(10.8)$ & $9(11.8)$ & $14(14.9)$ & $\begin{array}{l}239 \\
(11.5)\end{array}$ & $56(9.0)$ & $\begin{array}{l}19 \\
(11.4)\end{array}$ & $\begin{array}{l}10 \\
(14.1)\end{array}$ & $\begin{array}{l}85 \\
(9.9)\end{array}$ \\
\hline \multicolumn{11}{|c|}{ Concomitant medication at baseline ${ }^{c}, \mathrm{n}(\%)$} \\
\hline $\begin{array}{l}\text { Steroid, hormonal } \\
\text { drug }\end{array}$ & $\begin{array}{l}1118 \\
(37.9)\end{array}$ & $383(35.9)$ & $353(41.5)$ & $27(35.5)$ & $40(42.6)$ & $\begin{array}{l}803 \\
(38.5)\end{array}$ & $\begin{array}{l}229 \\
(36.8)\end{array}$ & $\begin{array}{l}55 \\
(33.1)\end{array}$ & $\begin{array}{l}31 \\
(43.7)\end{array}$ & $\begin{array}{l}315 \\
(36.7)\end{array}$ \\
\hline Anxiolytics & $\begin{array}{l}1427 \\
(48.4)\end{array}$ & $521(48.8)$ & $361(42.5)$ & $39(51.3)$ & $47(50.0)$ & $\begin{array}{l}968 \\
(46.4)\end{array}$ & $\begin{array}{l}319 \\
(51.3)\end{array}$ & $\begin{array}{l}96 \\
(57.8)\end{array}$ & $\begin{array}{l}44 \\
(62.0)\end{array}$ & $\begin{array}{l}459 \\
(53.4)\end{array}$ \\
\hline $\begin{array}{l}\text { Leukotriene } \\
\text { receptor } \\
\text { antagonists }\end{array}$ & $\begin{array}{l}173 \\
(5.9)\end{array}$ & $60(5.6)$ & $58(6.8)$ & $5(6.6)$ & $6(6.4)$ & $\begin{array}{l}129 \\
(6.2)\end{array}$ & $27(4.3)$ & $12(7.2)$ & $5(7.0)$ & $\begin{array}{l}44 \\
(5.1)\end{array}$ \\
\hline $\begin{array}{l}\text { Neurological } \\
\text { medication, } \\
\text { psychotropic drug }\end{array}$ & $\begin{array}{l}260 \\
(8.8)\end{array}$ & $92(8.6)$ & $54(6.4)$ & $7(9.2)$ & $6(6.4)$ & $\begin{array}{l}159 \\
(7.6)\end{array}$ & $60(9.6)$ & $\begin{array}{l}24 \\
(14.5)\end{array}$ & $\begin{array}{l}17 \\
(23.9)\end{array}$ & $\begin{array}{l}101 \\
(11.8)\end{array}$ \\
\hline $\begin{array}{l}\text { Analgesic, anti- } \\
\text { inflammatory }\end{array}$ & $\begin{array}{l}96 \\
(3.3)\end{array}$ & $36(3.4)$ & $26(3.1)$ & $2(2.6)$ & $1(1.1)$ & $\begin{array}{l}65 \\
(3.1)\end{array}$ & $22(3.5)$ & $9(5.4)$ & 0 & $\begin{array}{l}31 \\
(3.6)\end{array}$ \\
\hline $\begin{array}{l}\text { Antibacterial and/ } \\
\text { or antifungal }\end{array}$ & $\begin{array}{l}115 \\
(3.9)\end{array}$ & $34(3.2)$ & $33(3.9)$ & $2(2.6)$ & $6(6.4)$ & $\begin{array}{l}75 \\
(3.6)\end{array}$ & $27(4.3)$ & $9(5.4)$ & $4(5.6)$ & $\begin{array}{l}40 \\
(4.7)\end{array}$ \\
\hline $\begin{array}{l}\text { Antihypertensive, } \\
\text { cardiotonic }\end{array}$ & $\begin{array}{l}82 \\
(2.8)\end{array}$ & $26(2.4)$ & $26(3.1)$ & $2(2.6)$ & $2(2.1)$ & $\begin{array}{l}56 \\
(2.7)\end{array}$ & $17(2.7)$ & $7(4.2)$ & $2(2.8)$ & $\begin{array}{l}26 \\
(3.0)\end{array}$ \\
\hline Antipsychotic & $\begin{array}{l}607 \\
(20.6)\end{array}$ & $222(20.8)$ & $89(10.5)$ & $10(13.2)$ & $16(17.0)$ & $\begin{array}{l}337 \\
(16.1)\end{array}$ & $\begin{array}{l}178 \\
(28.6)\end{array}$ & $\begin{array}{l}59 \\
(35.5)\end{array}$ & $\begin{array}{l}33 \\
(46.5)\end{array}$ & $\begin{array}{l}270 \\
(31.4)\end{array}$ \\
\hline $\begin{array}{l}\text { Antismoking } \\
\text { product }\end{array}$ & $\begin{array}{l}17 \\
(0.6)\end{array}$ & $3(0.6)$ & $4(0.5)$ & 0 & $1(1.1)$ & $8(0.4)$ & $4(0.6)$ & $4(2.4)$ & $1(1.4)$ & $9(1.0)$ \\
\hline Antineoplastic & $\begin{array}{l}12 \\
(0.4)\end{array}$ & $4(0.4)$ & $4(0.5)$ & $1(1.3)$ & $1(1.1)$ & $\begin{array}{l}10 \\
(0.5)\end{array}$ & $1(0.2)$ & 0 & $1(1.4)$ & $2(0.2)$ \\
\hline Mood stabilizer & $\begin{array}{l}215 \\
(7.3)\end{array}$ & $63(5.9)$ & $27(3.2)$ & $5(6.6)$ & $4(4.3)$ & $\begin{array}{l}99 \\
(4.7)\end{array}$ & $\begin{array}{l}73 \\
(11.7)\end{array}$ & $\begin{array}{l}26 \\
(15.7)\end{array}$ & $\begin{array}{l}17 \\
(23.9)\end{array}$ & $\begin{array}{l}116 \\
(13.5)\end{array}$ \\
\hline Immunostimulant & $1(0)$ & $1(0.1)$ & 0 & 0 & 0 & $1(0.0)$ & 0 & 0 & 0 & 0 \\
\hline $\begin{array}{l}\text { Centrally-acting } \\
\text { anti-obesity } \\
\text { product }\end{array}$ & $2(0.1)$ & 0 & 0 & 0 & 0 & 0 & $1(0.2)$ & $1(0.6)$ & 0 & $2(0.2)$ \\
\hline Other & $\begin{array}{l}231 \\
(7.8)\end{array}$ & $80(7.5)$ & $68(8.0)$ & $4(5.3)$ & $7(7.4)$ & $\begin{array}{l}159 \\
(7.6)\end{array}$ & $48(7.7)$ & $\begin{array}{l}19 \\
(11.4)\end{array}$ & $5(7.0)$ & $\begin{array}{l}72 \\
(8.4)\end{array}$ \\
\hline $\begin{array}{l}\text { Sedative } \\
\text { antidepressant, n } \\
\text { (\%) }\end{array}$ & $\begin{array}{l}917 \\
(31.1)\end{array}$ & $288(27.0)$ & $251(29.5)$ & $26(34.2)$ & $38(40.4)$ & $\begin{array}{l}603 \\
(28.9)\end{array}$ & $\begin{array}{l}221 \\
(35.5)\end{array}$ & $\begin{array}{l}65 \\
(39.2)\end{array}$ & $\begin{array}{l}28 \\
(39.4)\end{array}$ & $\begin{array}{l}314 \\
(36.6)\end{array}$ \\
\hline Mirtazapine & 600 & $177(16.6)$ & $165(19.4)$ & $20(26.3)$ & $26(27.7)$ & 388 & 149 & 43 & 20 & 212 \\
\hline
\end{tabular}


Table 1 Patient demographics and clinical characteristics (Continued)

\begin{tabular}{|c|c|c|c|c|c|c|c|c|c|c|}
\hline \multirow[t]{2}{*}{ Variable } & \multirow{2}{*}{$\begin{array}{l}\text { All } \\
(N= \\
2946)\end{array}$} & \multicolumn{5}{|l|}{ Monotherapy } & \multicolumn{4}{|c|}{ Combination therapy } \\
\hline & & $\begin{array}{l}\text { Benzodiazepine } \\
(n=1067)\end{array}$ & $\begin{array}{l}\text { Non- } \\
\text { benzodiazepine } \\
(n=850)\end{array}$ & $\begin{array}{l}\text { Melatonin } \\
\text { receptor } \\
\text { agonist }(n= \\
76)\end{array}$ & $\begin{array}{l}\text { Orexin } \\
\text { receptor } \\
\text { antagonist } \\
(n=94)\end{array}$ & $\begin{array}{l}\text { Total } \\
(n= \\
2087)\end{array}$ & $\begin{array}{l}2 \\
\text { drugs } \\
(n= \\
622)\end{array}$ & $\begin{array}{l}3 \\
\text { drugs } \\
(n= \\
166)\end{array}$ & $\begin{array}{l}\geq 4 \\
\text { drugs } \\
(n= \\
71)\end{array}$ & $\begin{array}{l}\text { Total } \\
(n= \\
859)\end{array}$ \\
\hline & $(20.4)$ & & & & & $(18.6)$ & $(24.0)$ & $(25.9)$ & $(28.2)$ & $(24.7)$ \\
\hline $\begin{array}{l}\text { Trazodone } \\
\text { hydrochloride }\end{array}$ & $\begin{array}{l}360 \\
(12.2)\end{array}$ & $122(11.4)$ & $87(10.2)$ & $10(13.2)$ & $12(12.8)$ & $\begin{array}{l}231 \\
(11.1)\end{array}$ & $\begin{array}{l}88 \\
(14.1)\end{array}$ & $\begin{array}{l}27 \\
(16.3)\end{array}$ & $\begin{array}{l}14 \\
(19.7)\end{array}$ & $\begin{array}{l}129 \\
(15.0)\end{array}$ \\
\hline $\begin{array}{l}\text { Mianserin } \\
\text { hydrochloride }\end{array}$ & $\begin{array}{l}95 \\
(3.2)\end{array}$ & $31(2.9)$ & $19(2.2)$ & $1(1.3)$ & $4(4.3)$ & $\begin{array}{l}55 \\
(2.6)\end{array}$ & $28(4.5)$ & $8(4.8)$ & $4(5.6)$ & $\begin{array}{l}40 \\
(4.7)\end{array}$ \\
\hline \multicolumn{11}{|c|}{ Antidepressant dosage at end of treatment, $n(\%)$} \\
\hline $\begin{array}{l}\text { < Recommended } \\
\text { maximum dose }\end{array}$ & $\begin{array}{l}2740 \\
(93.0)\end{array}$ & $987(92.5)$ & $806(94.8)$ & $70(92.1)$ & $91(96.8)$ & $\begin{array}{l}1954 \\
(93.6)\end{array}$ & $\begin{array}{l}570 \\
(91.6)\end{array}$ & $\begin{array}{l}150 \\
(90.4)\end{array}$ & $\begin{array}{l}66 \\
(93.0)\end{array}$ & $\begin{array}{l}786 \\
(91.5)\end{array}$ \\
\hline $\begin{array}{l}\geq \text { Recommended } \\
\text { maximum dose }\end{array}$ & $\begin{array}{l}206 \\
(7.0)\end{array}$ & $80(7.5)$ & $44(5.2)$ & $6(7.9)$ & $3(3.2)$ & $\begin{array}{l}133 \\
(6.4)\end{array}$ & $52(8.4)$ & $16(9.6)$ & $5(7.0)$ & $\begin{array}{l}73 \\
(8.5)\end{array}$ \\
\hline \multicolumn{11}{|c|}{ Dosage of antidepressants during treatment $(\mathrm{mg} / \mathrm{day})^{\mathrm{d}}, \mathrm{n}(\%)$} \\
\hline Mean $(95 \% \mathrm{Cl})$ & $\begin{array}{l}74.3 \\
(72.3- \\
76.3)\end{array}$ & $73.0(69.9-76.1)$ & $68.2(64.8-71.7)$ & $67.3(52.9-81.7)$ & $69.1(57.9-80.4)$ & $\begin{array}{l}70.7 \\
(68.4- \\
72.9)\end{array}$ & $\begin{array}{l}82.5 \\
(77.4- \\
87.7)\end{array}$ & $\begin{array}{l}87.7 \\
(77.7- \\
97.7)\end{array}$ & $\begin{array}{l}77.4 \\
(66.6- \\
88.3)\end{array}$ & $\begin{array}{l}83.1 \\
(78.8- \\
87.4)\end{array}$ \\
\hline Median (IQR) & $\begin{array}{l}64.9 \\
(37.5 \\
100.0)\end{array}$ & $65.5(37.5,100.0)$ & $58.4(37.5,81.2)$ & $46.4(37.5,75.0)$ & $60.2(30.0 .85 .7)$ & $\begin{array}{l}61.1 \\
(37.5, \\
93.5)\end{array}$ & $\begin{array}{l}71.0 \\
(37.5 \\
105.1)\end{array}$ & $\begin{array}{l}75.0 \\
(37.5 \\
120.1)\end{array}$ & $\begin{array}{l}75.0 \\
(38.1 \\
105.0)\end{array}$ & $\begin{array}{l}73.0 \\
(37.5 \\
106.8)\end{array}$ \\
\hline$<75 \mathrm{mg} /$ day & $\begin{array}{l}1678 \\
(57.0)\end{array}$ & $605(56.7)$ & $526(61.9)$ & $53(69.7)$ & $54(57.4)$ & $\begin{array}{l}1238 \\
(59.3)\end{array}$ & $\begin{array}{l}328 \\
(52.7)\end{array}$ & $\begin{array}{l}80 \\
(48.2)\end{array}$ & $\begin{array}{l}32 \\
(45.1)\end{array}$ & $\begin{array}{l}440 \\
(51.2)\end{array}$ \\
\hline $\begin{array}{l}\geq 75 \text { and }<150 \\
\mathrm{mg} / \text { day }\end{array}$ & $\begin{array}{l}1005 \\
(34.1)\end{array}$ & $378(35.4)$ & $264(31.1)$ & $16(21.1)$ & $32(34.0)$ & $\begin{array}{l}690 \\
(33.1)\end{array}$ & $\begin{array}{l}218 \\
(35.0)\end{array}$ & $\begin{array}{l}62 \\
(37.3)\end{array}$ & $\begin{array}{l}35 \\
(49.3)\end{array}$ & $\begin{array}{l}315 \\
(36.7)\end{array}$ \\
\hline$\geq 150$ mg/day & $\begin{array}{l}263 \\
(8.9)\end{array}$ & $84(7.9)$ & $60(7.1)$ & $7(9.2)$ & $8(8.5)$ & $\begin{array}{l}159 \\
(7.6)\end{array}$ & $\begin{array}{l}76 \\
(12.2)\end{array}$ & $\begin{array}{l}24 \\
(14.5)\end{array}$ & $4(5.6)$ & $\begin{array}{l}104 \\
(12.1)\end{array}$ \\
\hline
\end{tabular}

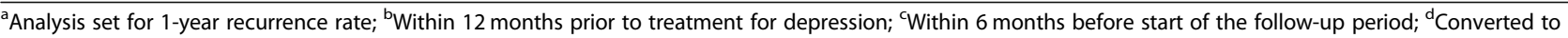
values that are equivalent to imipramine using a dose-equivalence scale for antidepressants [20].

$A D H D$ Attention deficit hyperactivity disorder, $\mathrm{Cl}$ Confidence interval, IQR Interquartile range, SD Standard deviation

quarters of patients with MDD are prescribed multiple therapies [9]. Benzodiazepines formed the backbone of antidepressant therapy and combination therapy in this study, in concordance with benzodiazepine monotherapy being the preferred initial treatment for MDD among Japanese clinicians [24], and a combination of first- and/ or second-generation antidepressants and benzodiazepines were the predominant polypharmacy [9].

That hypnotic therapy with two drugs was more common than $\geq 3$-drug regimens is perhaps not surprising, and may reflect the reduced payments from public insurance payers to medical institutions if $\geq 3$ hypnotics are simultaneously prescribed [22]. Likewise, our observation that almost all patients were prescribed less than the recommended maximum dose of antidepressants is in keeping with prescribing practices in Japan [22].

Therefore, if depression-associated insomnia can be effectively managed using fewer medications, without increasing the risk of depression recurrence, then combination therapy - with its potential for drug-drug interactions - should be avoided whenever possible. Furthermore, consideration should be given to the hypnotic prescribed for depression-associated insomnia because efficacy and safety varies across classes. For example, benzodiazepines are associated with multiple adverse events, including cognitive and psychomotor impairment, amnesia, next-day hangover, rebound insomnia and dependence [13]. In contrast, newer hypnotic therapies including melatonin agonists, improve sleep without producing residual sedation, or memory, cognitive or psychomotor impairment [13]. In this study, no difference in MDD recurrence was found by hypnotic drug type, suggesting that these advantages may be realized without increasing risk of depression relapse in patients with residual insomnia. However, a recent meta-analysis has reported differences in MDD relapse rates according to class of antidepressant therapy [25].

This study is limited by the JMDC database only providing data for employees aged 20-64 years and their spouse or family members, and the majority of study subjects $(62.8 \%)$ were male, meaning the results may not be generalizable to the overall Japanese population. Furthermore, the database does not capture the criteria used to diagnose patients with MDD and the severity of depression, which could influence diagnostic and prescribing patterns. Likewise, patients with severe depression 


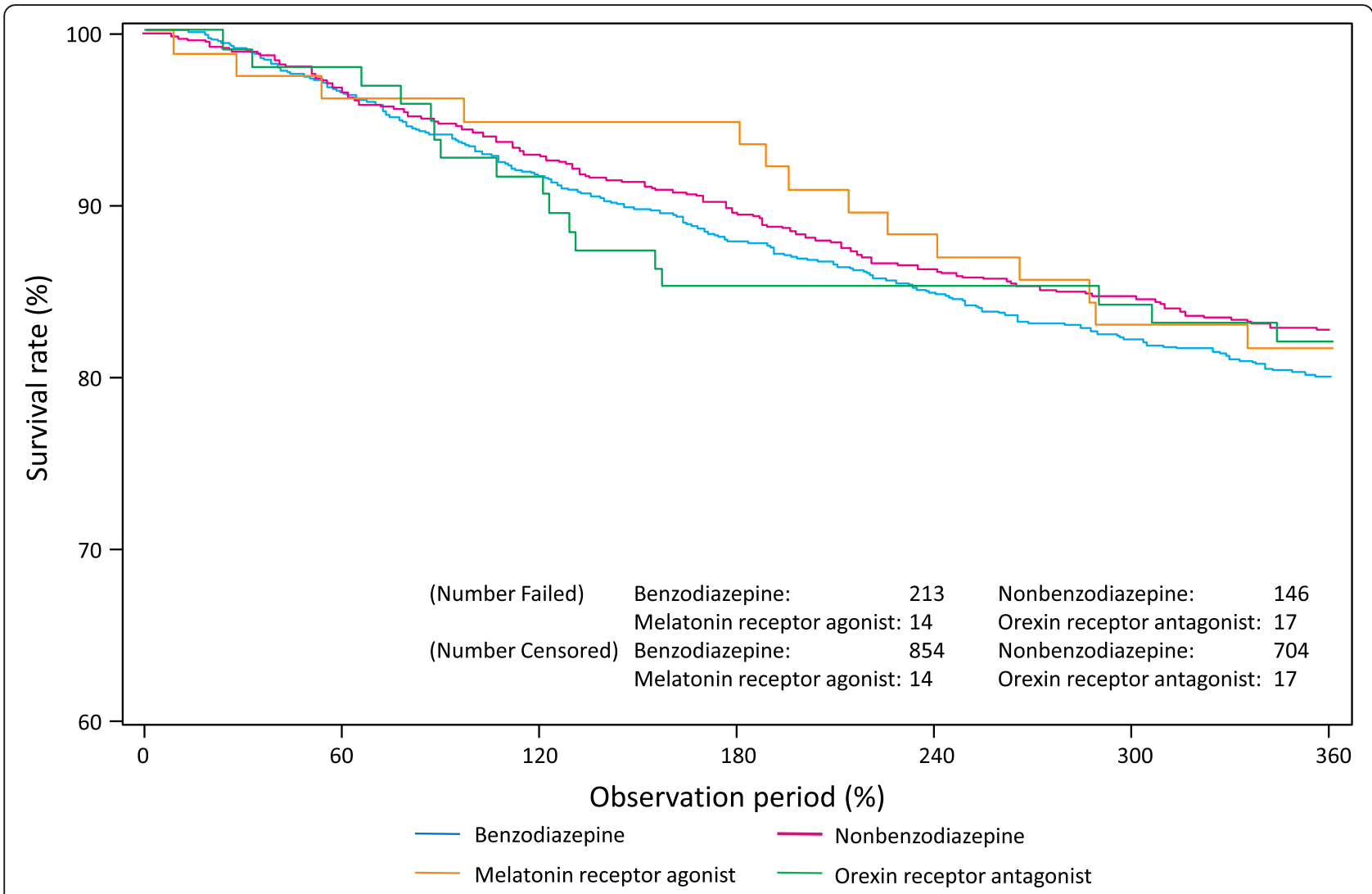

Fig. 3 Time to MDD recurrence in patients prescribed hypnotic monotherapy for residual insomnia. MDD, major depressive disorder

have a higher risk of being unemployed, and would therefore not be captured in the JMDC database [26], which could be evidenced by the higher odds of recurrence in spouses and other family members versus insured employees observed in this study.

Similarly, a filled prescription claim does not indicate consumption of, or adherence to, prescription medication and it was assumed that patients who discontinued antidepressant treatment were in remission, as opposed to failing to return to a healthcare provider to refill their prescription. Likewise, it was inferred that patients were prescribed hypnotic therapy during follow-up to manage depression-associated residual insomnia.

\section{Conclusions}

The rates of MDD recurrence in patients receiving hypnotics for residual insomnia was not affected by hypnotic monotherapy versus combination therapy, or class of hypnotic therapy, indicating that choice of ongoing treatment for insomnia is unlikely to increase the risk of recurrent MDD once antidepressant therapy ceases for real-world Japanese patients. Benzodiazepine monotherapy is the most commonly prescribed hypnotic among Japanese patients with MDD, though a substantial proportion of patients are prescribed $\geq 2$ hypnotic therapies.
Hypnotics should be appropriately prescribed for depression-associated insomnia, given differences in efficacy and safety across classes of hypnotics. Newer classes of hypnotics may be useful for avoiding some of the drawbacks of benzodiazepine therapy.

\section{Abbreviations}

ATC: Anatomical Therapeutic Chemical; Cl: Confidence interval; ICD10: International Statistical Classification of Diseases and Related Health Problems (10th ed.); IQR: Interquartile range; JMDC: Japan Medical Data Center; MDD: Major depressive disorder; OR: Odds ratio; SD: Standard deviation; SNRI: Serotonin-noradrenaline reuptake inhibitor; SSRI: Selective serotonin reuptake inhibitor

\section{Acknowledgements}

The authors are grateful to Chie Ito, MSc and Yoshimitsu Takamatsu, MEc (JMDC Inc., Tokyo, Japan), sponsored by Takeda Pharmaceutical Company Limited, for support with designing the conceptual idea and implementation plan of the study, statistical analysis and data management. The authors would like to acknowledge the writing support of MIMS (Hong Kong) Ltd., during the development of this paper, which was funded by Takeda Pharmaceutical Company Limited, in compliance with Good Publication Practice 3 ethical guidelines (Battisti WP et al. Ann Intern Med 2015;163:4614).

\section{Authors' contributions}

All authors contributed to the conception of the study design; KY, IK, EM $\mathrm{TM}, \mathrm{MT}$ and KM to the data analysis and interpretation; KY, Kl, ME, TM, MT and $\mathrm{KM}$ to drafting of the manuscript; and $\mathrm{KY}, \mathrm{KI}, \mathrm{ME}, \mathrm{TM}$ and $\mathrm{KM}$ to revising the manuscript for important intellectual content. All authors reviewed and 
Table 2 Multivariate analysis for predictors of MDD recurrence

\begin{tabular}{|c|c|c|c|}
\hline Variable & Reference & Category & $\begin{array}{l}\text { MDD recurrence at } 360 \text { days, OR } \\
(95 \% \mathrm{Cl})\end{array}$ \\
\hline \multirow[t]{6}{*}{ Prescription pattern } & \multirow[t]{6}{*}{ Benzodiazepine } & Non-benzodiazepine & $0.84(0.67-1.07)$ \\
\hline & & Melatonin receptor agonist & $0.87(0.47-1.60)$ \\
\hline & & Orexin receptor antagonist & $0.87(0.50-1.50)$ \\
\hline & & $\begin{array}{l}\text { Combination therapy }(2 \\
\text { drugs) }\end{array}$ & $1.23(0.97-1.56)$ \\
\hline & & $\begin{array}{l}\text { Combination therapy ( } 3 \\
\text { drugs) }\end{array}$ & $0.91(0.60-1.37)$ \\
\hline & & $\begin{array}{l}\text { Combination therapy ( } \geq 4 \\
\text { drugs) }\end{array}$ & $0.97(0.53-1.75)$ \\
\hline Gender $^{\mathrm{a}}$ & Male & Female & $0.67(0.50-0.88)$ \\
\hline $\mathrm{Age}^{\mathrm{a}}$ & $<40$ years & $\geq 40$ years & $0.95(0.78-1.14)$ \\
\hline \multirow[t]{2}{*}{ Insured or dependent } & \multirow[t]{2}{*}{ Insured } & Spouse & $1.44(1.03-2.02)$ \\
\hline & & Other family member & $1.46(0.99-2.16)$ \\
\hline $\begin{array}{l}\text { Prior neurological medication, psychotropic } \\
\text { drug }\end{array}$ & Absent & Present & $1.22(0.86-1.73)$ \\
\hline Prior anxiolytic medication & Absent & Present & $0.98(0.77-1.24)$ \\
\hline Concomitant antipsychotic medication & Absent & Present & $1.11(0.88-1.39)$ \\
\hline Sedative antidepressant & Absent & Present & $1.50(1.24-1.82)$ \\
\hline Antidepressant dosage at end of treatment & $\begin{array}{l}<\text { Recommended maximum } \\
\text { dose }\end{array}$ & $\begin{array}{l}\geq \text { Recommended maximum } \\
\text { dose }\end{array}$ & $1.11(0.77-1.60)$ \\
\hline \multirow[t]{2}{*}{ Antidepressant dosage $(\mathrm{mg} / \text { day })^{\mathrm{b}}$} & \multirow[t]{2}{*}{$<75 \mathrm{mg} /$ day } & $\geq 75$ and $<150 \mathrm{mg} /$ day & $1.06(0.86-1.29)$ \\
\hline & & $\geq 150$ mg/day & $1.14(0.81-1.61)$ \\
\hline
\end{tabular}

${ }^{\mathrm{a}}$ Mandatory variables; ${ }^{\mathrm{b}}$ Converted to values that are equivalent to imipramine using a dose-equivalence scale for antidepressants [20].

Cl Confidence interval, MDD Major depressive disorder, OR Odds ratio

approved the final draft of this manuscript and agree to be accountable for all aspects of the work.

\section{Funding}

This study was funded by Takeda Pharmaceutical Company Limited. Takeda Pharmaceutical Company Limited participated in the design of the study, data collection and analysis, interpretation of results and the preparation of the manuscript.

\section{Availability of data and materials}

The data set used for analysis in this study is available from JMDC Inc. but were used under license for the current study. Restrictions thus apply, and the data are not publicly available. For inquiries about access to the data set used in this study, please contact JMDC (https://www.jmdc.co.jp).

\section{Ethics approval and consent to participate}

This study used anonymized data. Because the study used anonymized information, institutional ethics approval and informed consent were not required, in accordance with Ethical Guidelines for Medical and Health Research Involving Human Subjects in Japan.

\section{Consent for publication}

Not applicable.

\section{Competing interests}

Dr. Inada received personal fees from Dainippon Sumitomo Pharma, Eisai, Eli Lilly Japan, Janssen pharmaceutical, Meiji-Seika Pharmaceutical, Mochida, MSD, Novartis, Otsuka Pharmaceutical, Shionogi, Tanabe-Mitsubishi Pharma and Yoshitomi Yakuhin, and grants from MSD, outside the submitted work. Dr. Enomoto has no conflict of interest to disclose. Mr. Yamato and Mr. Marumoto are employed by Takeda Pharmaceutical Company Limited. Dr. Takeshima received lecture fees from Daiichi Sankyo Company outside the submitted work. Prof Mishima received speaker's honoraria from Eisai Co.,
Ltd., MSD Inc., Takeda Pharmaceutical Co., Ltd., Astellas Pharma Inc., Pfizer Inc., Otsuka Pharmaceutical Co., Ltd., Mochida Pharmaceutical Co., Ltd., Mitsubishi Tanabe Pharma Corporation (Yoshitomiyakuhin Corporation) and Janssen Pharmaceutical, consulting fee from Taisho Pharmaceutical Co., Ltd., research support from the Japanese Ministry of Health, Labour and Welfare (H29-Seishin-Ippan-001, 19GC1012), the Japanese Ministry of Education, Science, and Technology and the National Center of Neurology and Psychiatry (Intramural Research Grant for Neurological and Psychiatric Disorders), and research grants from Eisai Co., Ltd., Nobelpharma Co., Ltd., Otsuka Pharmaceutical Co., Ltd. and Takeda Pharmaceutical Co., Ltd.

\section{Author details}

'Japan Medical Office, Takeda Pharmaceutical Company Limited, Tokyo, Japan. ²Department of Psychiatry, Tokyo Women's Medical University, Tokyo, Japan. ${ }^{3}$ Department of Medical Technology, School of Health Sciences, Tokyo University of Technology, Tokyo, Japan. ${ }^{4}$ Department of Neuropsychiatry, Akita University Graduate School of Medicine, Akita, Japan. ${ }^{5}$ Department of Psychophysiology, National Institute of Mental Health, National Center of Neurology and Psychiatry, Tokyo, Japan. International Institute for Integrative Sleep Medicine, Tsukuba, Japan.

Received: 30 October 2020 Accepted: 5 January 2021

Published online: 13 January 2021

\section{References}

1. Ishikawa H, Kawakami N, Kessler RC. Lifetime and 12-month prevalence, severity and unmet need for treatment of common mental disorders in Japan: results from the final dataset of world mental health Japan survey. Epidemiol Psychiatr Sci. 2016;25(3):217-29.

2. Ishikawa H, Tachimori H, Takeshima T, Umeda M, Miyamoto K, Shimoda H, et al. Prevalence, treatment, and the correlates of common mental disorders in the mid 2010's in Japan: the results of the world mental health Japan 2nd survey. J Affect Disord. 2018;241:554-62. 
3. Naganuma $Y$, Tachimori H, Kawakami N, Takeshima T, Ono Y, Uda H, et al. Twelve-month use of mental health services in four areas in Japan: findings from the world mental health Japan survey 2002-2003. Psychiatry Clin Neurosci. 2006;60(2):240-8.

4. GBD 2016 Disease and Injury Incidence and Prevalence Collaborators. Global, regional, and national incidence, prevalence, and years lived with disability for 328 diseases and injuries for 195 countries, 1990-2016: a systematic analysis for the global burden of disease study 2016. Lancet. 2017:390(10100):1211-59.

5. Evans-Lacko S, Knapp M. Global patterns of workplace productivity for people with depression: absenteeism and presenteeism costs across eight diverse countries. Soc Psychiatry Psychiatr Epidemiol. 2016;51(11):1525-37.

6. Okumura Y, Higuchi T. Cost of depression among adults in Japan. Prim Care Companion CNS Disord. 2011;13(3):PCC.10m01082.

7. Asami Y, Goren A, Okumura Y. Work productivity loss with depression, diagnosed and undiagnosed, among workers in an internet-based survey conducted in Japan. J Occup Environ Med. 2015;57(1):105-10.

8. American Psychiatric Association. Practice guidelines for the treatment of patients with major depressive disorder. Third edition. Available at: https:// psychiatryonline.org/pb/assets/raw/sitewide/practice_guidelines/guidelines/ mdd.pdf. Accessed 8 September 2020.

9. Onishi Y, Hinotsu S, Furukawa TA, Kawakami K. Psychotropic prescription patterns among patients diagnosed with depressive disorder based on claims database in Japan. Clin Drug Investig. 2013;33(8):597-605.

10. Hiranyatheb T, Nakawiro D, Wongpakaran T, Wongpakaran N, Bookkamana $P$, Pinyopornpanish $M$, et al. The impact of residual symptoms on relapse and quality of life among Thai depressive patients. Neuropsychiatr Dis Treat. 2016;12:3175-81.

11. Buckman JEJ, Underwood A, Clarke K, Saunders R, Hollon SD, Fearon P, et al. Risk factors for relapse and recurrence of depression in adults and how they operate: a four-phase systematic review and meta-synthesis. Clin Psychol Rev. 2018:64:13-38.

12. Sakurai H, Suzuki T, Yoshimura K, Mimura M, Uchida H. Predicting relapse with individual residual symptoms in major depressive disorder: a reanalysis of the STAR*D data. Psychopharmacology. 2017;234(16):2453-61.

13. Srinivasan V, Brzezinski A, Pandi-Perumal SR, Spence DW, Cardinali DP, Brown GM. Melatonin agonists in primary insomnia and depressionassociated insomnia: are they superior to sedative-hypnotics? Prog NeuroPsychopharmacol Biol Psychiatry. 2011;35(4):913-23.

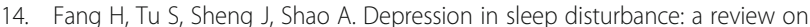
a bidirectional relationship, mechanisms and treatment. J Cell Mol Med. 2019;23(4):2324-32.

15. Wichniak A, Wierzbicka A, Walęcka M, Jernajczyk W. Effects of antidepressants on sleep. Curr Psychiatry Rep. 2017;19(9):63.

16. Asnis GM, Chakraburtty A, DuBoff EA, Krystal A, Londborg PD, Rosenberg R, et al. Zolpidem for persistent insomnia in SSRI-treated depressed patients. J Clin Psychiatry. 1999;60(10):668-76.

17. Krystal A, Fava M, Rubens R, Wessel T, Caron J, Wilson P, et al. Evaluation of eszopiclone discontinuation after cotherapy with fluoxetine for insomnia with coexisting depression. J Clin Sleep Med. 2007;3(1):48-55.

18. Chung KH, Li CY, Kuo SY, Sithole T, Liu WW, Chung MH. Risk of psychiatric disorders in patients with chronic insomnia and sedative-hypnotic prescription: a nationwide population-based follow-up study. J Clin Sleep Med. 2015;11(5):543-51.

19. Li CT, Bai YM, Lee YC, Mao WC, Chen MH, Tu PC, et al. High dosage of hypnotics predicts subsequent sleep-related breathing disorders and is associated with worse outcomes for depression. Sleep. 2014;37(4):803-9.

20. Inagaki A, Inada T. Dose equivalence of psychotropic drugs. Part XXVII: dose equivalence of novel antidepressants II: venlafaxine. Jpn J Clin Psychopharmacol. 2018;21:547-62.

21. Melartin TK, Rytsälä HJ, Leskelä US, Lestelä-Mielonen PS, Sokero TP, Isometsä ET. Severity and comorbidity predict episode duration and recurrence of DSM-IV major depressive disorder. J Clin Psychiatry. 2004;65(6):810-9.

22. Inada T, Inagaki A. Psychotropic dose equivalence in Japan. Psychiatry Clin Neurosci. 2015;69(8):440-7.

23. Mahlich J, Tsukazawa S, Wiegand F. Estimating prevalence and healthcare utilization for treatment-resistant depression in Japan: a retrospective claims database study. Drugs Real World Outcomes. 2018;5(1):35-43.

24. Nakagawa A, Williams A, Sado M, Oguchi Y, Mischoulon D, Smith F, et al. Comparison of treatment selections by Japanese and US psychiatrists for major depressive disorder: a case vignette study. Psychiatry Clin Neurosci. 2015;69(9):553-62.

25. Kato $M$, Hori H, Inoue T, Iga J, Iwata M, Inagaki T, et al. Discontinuation of antidepressants after remission with antidepressant medication in major depressive disorder: a systematic review and meta-analysis. Mol Psychiatry. 2020. https://doi.org/10.1038/s41380-020-0843-0 [Epub ahead of print].

26. Rizvi SJ, Cyriac A, Grima E, Tan M, Lin P, Gallaugher LA, et al. Depression and employment status in primary and tertiary care settings. Can J Psychiatr. 2015;60(1):14-22.

\section{Publisher's Note}

Springer Nature remains neutral with regard to jurisdictional claims in published maps and institutional affiliations.
Ready to submit your research? Choose BMC and benefit from:

- fast, convenient online submission

- thorough peer review by experienced researchers in your field

- rapid publication on acceptance

- support for research data, including large and complex data types

- gold Open Access which fosters wider collaboration and increased citations

- maximum visibility for your research: over $100 \mathrm{M}$ website views per year

At BMC, research is always in progress.

Learn more biomedcentral.com/submissions 